\title{
Avaliação da emissão de formaldeído em compósitos fabricados com adição de cascas de Pinus caribaea var. caribaea
}

\author{
Maria Vanessa Egger Schueler, Roberto Carlos Costa Lelis, Alexandre Miguel do \\ Nascimento, Fernanda Lago Morbeck, Rayssa de Medeiros Morais, Gregório Mateus Santana
}

Universidade Federal Rural do Rio de Janeiro, Seropédica, Rio de Janeiro, Brasil.

\begin{abstract}
RESUMO Com a crescente preocupação com relação à emissão de formaldeído em ambientes internos dada a sua periculosidade à saúde humana, o presente trabalho teve por objetivo avaliar o emprego de cascas de Pinus caribaea var. caribaea para atenuar a emissão de formaldeído em compósitos aglomerados prensados sob diferentes temperaturas e proporções de casca. Os compósitos foram produzidos em duas temperaturas de prensagem $\left(140^{\circ} \mathrm{C}\right.$ e $\left.160^{\circ} \mathrm{C}\right)$, cinco proporções de partícula casca $\left(0,5,10,15\right.$ e 20\%), tempo de prensagem de 7 minutos, densidade nominal de $0,70 \mathrm{~g} \mathrm{~cm}^{-1}$, pressão específica

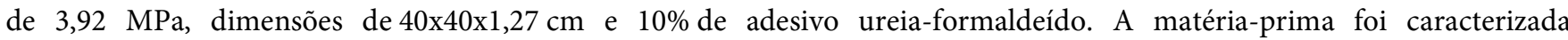
considerando a densidade básica e aparente e os compósitos produzidos caracterizados consoantes suas propriedades físicas (densidade, umidade, inchamento em espessura e absorção de água após 2 e 24 horas de imersão), propriedades mecânicas (ligação interna, módulo de ruptura - MOR - e módulo de elasticidade - MOE - à flexão estática) e químicas (pH, capacidade tampão e concentração de formaldeído). Os resultados mostraram a viabilidade de se produzir compósitos com cascas de Pinus caribaea var. caribaea bem como observou uma redução na emissão de formaldeído em razão do aumento da proporção de casca.
\end{abstract}

Palavras-chave: pinus, material lignocelulósico, emissão de formaldeído.

\section{Evaluation of the issue of formaldehyde in composites manufactured with added barks of Pinus caribaea var. caribaea}

\begin{abstract}
With the growing concern about the emission of formaldehyde in indoor environments in view of its health hazard, the present work aims to evaluate the use of bark of Pinus caribaea var. caribaea to attenuate the emission of formaldehyde in composites agglomerates pressed at different temperatures and bark proportions. The composites were produced at two pressing temperatures $\left(140^{\circ} \mathrm{C}\right.$ and $\left.160^{\circ} \mathrm{C}\right)$, five bark particle proportions $(0,5,10,15$ and $20 \%)$, pressing time of 7 minutes, nominal density of $0.70 \mathrm{~g} . \mathrm{cm}^{-1}$, specific pressure of $3.92 \mathrm{MPa}$, dimensions of 40x40x1.27 $\mathrm{cm}$ and $10 \%$ of ureaformaldehyde adhesive. The raw material was characterized considering the basic and apparent density and the agglomerates composites characterized according to their physical properties (density, humidity, swelling in thickness and water absorption after 2 and 24 hours of immersion), mechanical properties (internal bonding, modulus of rupture - MOR - and modulus of elasticity - MOE - to static bending) and chemical ( $\mathrm{pH}$, buffer capacity and formaldehyde concentration). The results showed the feasibility of producing composites with Pinus caribaea var. caribaea as well as to obtain a reduction in the emission of formaldehyde due to the increase in the proportion of bark.
\end{abstract}

Keywords: pine, lignocellulosic material, formaldehyde emission.

\section{Introdução}

O formaldeído é um dos principais compostos utilizados na fabricação de painéis de madeira, na colagem de madeira sólida, em compósitos laminados e particulados. Embora seja um contaminante de ambientes fechados, seu uso ainda é elevado nas indústrias (JANTUNEN et al., 2008). Segundo Dai et al. (2018) a exposição ao formaldeído mediante 
o contato regular em ambientes internos causa impactos negativos substanciais à saúde humana.

As pesquisas relacionadas à concentração de formaldeído em ambientes internos iniciaram no final dos anos 1980, seguindo as ações regulatórias propostas quanto as exposições residenciais e os efeitos associados à saúde humana. Por consequência, ao longo das últimas décadas, vários estudos relataram a presença de diversos níveis internos de formaldeído em edifícios e casas, em compostos de madeira como painéis, móveis e pisos (PIERCE et al., 2016).

Em locais fechados, o formaldeído apresenta aproximadamente $60 \%$ de sua totalidade constituído por aldeídos (PEREIRA et al., 2001). É também identificado como uma substância com alto poder cancerígeno em humanos, não existindo um grau seguro de exposição, consoante relata a International Agency for Research on Cancer - IARC (2006). A emissão de formaldeído é ocasionada pela quebra das ligações na resina pela alta exposição à umidade (CAMPOS; LAHR, 2004). Segundo He et al. (2012), essas emissões são fortemente influenciadas pela matéria-prima utilizada e também ao processo de fabricação dos painéis.

Em relação aos compósitos fabricados, estes são influenciados diretamente pelo tipo de matéria-prima empregada (densidade, características anatômicas, umidade, extrativos e $\mathrm{pH}$ da madeira) e pelo processo de fabricação. A taxa de compactação, o tipo de resina, tamanho e orientação das partículas, tempo de fechamento da prensa, temperatura de prensagem e pressão específica, todos esses fatores alteram a qualidade do compósito (MESQUITA et al., 2015).

A indústria madeireira e a exploração florestal geram uma elevada quantidade de resíduos, o que torna crescente o interesse pelo reaproveitamento dos mesmos sobretudo para a produção de compósitos aglomerados (BRITO et al., 2005). Os resíduos de casca, por exemplo, vêm sendo amplamente utilizados para a fabricação de compósitos. Desse modo, a casca representa uma alternativa promissora e sustentável para a produção de compósitos em conjunto com outras partículas de madeira (BRITO et al., 2005; TRIANOSKI et al., 2013).

Isto posto, o objetivo deste trabalho foi avaliar o emprego de cascas de Pinus caribaea var. caribaea na fabricação de compósitos aglomerados (prensados em diferentes temperaturas e proporções de casca) como elemento captador de formaldeído, para atenuar a sua emissão.

\section{Material e Métodos}

\section{Matéria-prima, obtenção das partículas e}

\section{densidade da madeira e da casca}

A madeira de Pinus caribaea var. caribaea foi obtida em um povoamento florestal, com idade estimada de 27 anos $($ DAP $=20 \mathrm{~cm})$, localizado no município de Seropédia, Rio de Janeiro. Já as cascas de Pinus caribaea var. caribaea foram coletadas de cinco árvores $(\mathrm{DAP}=15-40 \mathrm{~cm})$ com idade de 35 anos, escolhidas aleatoriamente, obtidas de dois talhões de uma empresa florestal, localizada no município de Estrela do Sul, Minas Gerais.

As árvores foram cortadas em toretes, sendo estes seccionados em discos de aproximadamente $5,0 \mathrm{~cm}$ de espessura, buscando de início a geração de partículas tipo strand (gerador de partículas) e logo em seguida tipo sliver (moinho de martelo). Após isso, esse material foi seco ao ar até atingir 13\% de umidade, quando então foi peneirado para retirada dos finos. Para a fabricação dos compósitos foram selecionadas as partículas que passaram pela peneira de 4,1 $\mathrm{mm}$ e ficaram retidas na de $0,49 \mathrm{~mm}$, sendo em seguida secas em estufa a $103 \pm 2{ }^{\circ} \mathrm{C}$ até atingirem uma umidade média de $6 \%$. Para as cascas, foram utilizadas partículas com espessura entre 2 a $4 \mathrm{~mm}$, sendo estas também obtidas utilizando 
moinho de martelo, e posteriormente secas em estufa à 103 \pm 2 ${ }^{\circ} \mathrm{C}$ até atingirem umidade média de $6 \%$.

Para a determinação da densidade básica da madeira e da casca de Pinus caribaea var. caribaea foi utilizado o método de deslocamento em mercúrio $(\mathrm{Hg})$ e para a determinação da densidade aparente foi utilizado o método de imersão em água, conforme Vital (1984).

\section{Produção dos compósitos}

Foram produzidos compósitos de média densidade, tipo P2, conforme - Norma Brasileira - NBR 14810 da Associação Brasileira de Normas Técnicas - ABNT (2013), classificados como compósitos não estruturais para uso interno em condições secas. Os seguintes parâmetros foram considerados na produção dos compósitos: (a) dimensões dos compósitos de 40x40x1,27 cm; (b) densidade nominal dos compósitos de $0,70 \mathrm{~g} \mathrm{~cm}^{-3}$; (c) pressão obtida na prensagem de 3,92 MPa; (d) tempo de fechamento de $45 \mathrm{~s}$; (e) umidade das partículas de 6\%; (f) conteúdo de resina (\% base peso seco) de 10\%; e (g) catalisador: solução de sulfato de amônia $\left(\mathrm{NH}_{4}\right)_{2} \mathrm{SO}_{4} 24 \%$ na razão de $2 \%$ sobre a massa total de sólidos da resina.

Para a fabricação foi utilizado um delineamento inteiramente casualizado com um arranjo fatorial de $5 \times 2$, isto é, contendo cinco proporções de madeira/casca e duas temperaturas de prensagem, com três repetições para cada tratamento, totalizando 30 compósitos (Tabela 1).

O adesivo foi aplicado em um tambor rotatório por aspersão, com o auxílio de uma pistola pneumática, promovendo a distribuição uniforme do adesivo sobre a superfície das partículas. Os colchões de partículas foram formados de maneira manual para evitar a perda de partículas (nas bordas) no momento do transporte para a prensa hidráulica. Depois de prontos, os mesmos foram climatizados a uma umidade relativa de $65 \pm 5 \%$ e temperatura de $20 \pm 3{ }^{\circ} \mathrm{C}$.
Os compósitos aglomerados foram então cortados de acordo com a NBR 14810 (ABNT, 2013) para obtenção dos corpos de prova e posterior realização dos ensaios tecnológicos.

Tabela 1. Delineamento experimental.

Table 1. Experimental design.

\begin{tabular}{|c|c|c|c|}
\hline \multirow{2}{*}{$\begin{array}{c}\text { Tratamentos } \\
\text { realizados }\end{array}$} & \multicolumn{2}{|c|}{$\begin{array}{l}\text { Composição do } \\
\text { compósito (\%) }\end{array}$} & \multirow{2}{*}{$\begin{array}{c}\text { Temperatura } \\
\text { de prensagem } \\
\left({ }^{\circ} \mathrm{C}\right)\end{array}$} \\
\hline & $\begin{array}{l}\text { Partícula } \\
\text { Madeira }\end{array}$ & $\begin{array}{c}\text { Partícula } \\
\text { Casca }\end{array}$ & \\
\hline $0 \mathrm{C} / 140$ & 100 & 0 & \\
\hline $5 \mathrm{C} / 140$ & 95 & 5 & \\
\hline $10 \mathrm{C} / 140$ & 90 & 10 & 140 \\
\hline $15 \mathrm{C} / 140$ & 85 & 15 & \\
\hline $20 \mathrm{C} / 140$ & 80 & 20 & \\
\hline $0 \mathrm{C} / 160$ & 100 & 0 & \\
\hline $5 \mathrm{C} / 160$ & 95 & 5 & \\
\hline $10 \mathrm{C} / 160$ & 90 & 10 & 160 \\
\hline $15 \mathrm{C} / 160$ & 85 & 15 & \\
\hline $20 \mathrm{C} / 160$ & 80 & 20 & \\
\hline
\end{tabular}

\section{Propriedades físicas e mecânicas}

As propriedades físicas e mecânicas dos compósitos aglomerados produzidos foram determinadas de acordo com a NBR 14810 (ABNT, 2013). Para os os ensaios físicos foram analisadas as propriedades tais como: (a) densidade dos compósitos, (b) teor de umidade, (c) inchamento em espessura (IE) após 2 e 24h e (g) a absorção de água (AA) após 2 e 24 h.

Em relação aos ensaios mecânicos, estes foram realizados em uma máquina universal de ensaios, sendo estudados: a resistência à flexão estática (Módulo de Ruptura - MOR e Módulo de Elasticidade - MOE) e de Ligação Interna - LI (Resistência à tração perpendicular ao plano dos compósitos). Foram feitos três compósitos de cada tratamento, e retirado de cada compósito quatro amostras para cada ensaio, totalizando assim doze repetições por ensaio.

\section{Propriedades químicas}

Foram obtidos particulados a partir dos compósitos, sendo utilizados $5 \mathrm{~g}$ (base seca) em $150 \mathrm{~mL}$ de água destilada, 
sob refluxo $\left(100^{\circ} \mathrm{C}\right)$ por 20 minutos para a determinação do $\mathrm{pH}$ (utilizando um pH-metro digital). Para a capacidade tampão, os extratos foram titulados com solução de $\mathrm{NaOH}$ $0,001 \mathrm{~N}$ até $\mathrm{pH} 7$, posteriormente transformados em mmol $\mathrm{NaOH} / 5 \mathrm{~g}$. A concentração de formaldeído foi determinada conforme a Norma Europeia - EN 717-3 (1996).

\section{Análises dos dados}

As variáveis dependentes foram analisadas sob o efeito do teor de partículas, a temperatura de prensagem e da interação entre os dois fatores. Os dados foram analisados no programa Statistica 7, atendendo as pressuposições da análise de variância pelo teste Hartley, Cochran C, Bartlett, normalidade e homogeneidade de variâncias pelo teste Shapiro-Wilk, e usado o Teste de Tukey ao nível de 5\% de significância. Não atendendo às pressuposições, foi aplicado o teste não paramétrico de Kruskal-Wallis para comparações de médias.

\section{Resultados e Discussão}

\section{Densidade básica e aparente da madeira e da}

\section{casca}

Os valores médios da densidade básica da madeira e da casca de Pinus caribaea var. caribaea foram de $0,54 \mathrm{~g} \mathrm{~cm}^{-3} \mathrm{e}$ $0,21 \mathrm{~g} \mathrm{~cm}^{-3}$, respectivamente, na faixa recomendada por Maloney (1993) para a produção de painéis de partículas até $0,60 \mathrm{~g} \mathrm{~cm}^{-3}$. A densidade aparente da madeira foi de $0,60 \mathrm{~g} \mathrm{~cm}^{-}$ ${ }^{3}$ e da casca $0,22 \mathrm{~g} \mathrm{~cm}^{-3}$; e a umidade de $12 \%$ para a madeira e 18,6\% para a casca. Carvalho et al., (2015) obtiveram um valor menor para a densidade básica de Pinus caribaea var. hondurensis, $0,49 \mathrm{~g} \mathrm{~cm}^{-3}$. Em relação a casca, Furtado et al., (2010) encontraram valores para Pinus spp entre 0,25 a 0,29 $\mathrm{g} \mathrm{cm}^{-3}$, superiores ao obtido neste estudo.

\section{Densidade e umidade dos compósitos}

Os valores de densidade das chapas prensadas na

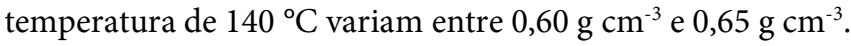
Para aquelas prensadas na temperatura de $160^{\circ} \mathrm{C}$, os valores se encontram na faixa entre $0,54 \mathrm{~g} \mathrm{~cm}^{-3}$ e $0,65 \mathrm{~g} \mathrm{~cm}^{-3}$ (Tabela 2). Ainda, as proporções de cascas empregadas não influenciaram diretamente na densidade dos compósitos, como pode ser verificado pela análise estatística entre os tratamentos. Carvalho et al., (2015) encontraram uma densidade de $0,67 \mathrm{~g} \mathrm{~cm}^{-3}$ para painéis confeccionados com apenas madeira de Pinus spp.

Pela análise de variância da densidade e umidade dos compósitos foi observado que o efeito da interação foi significativo, tendo em vista que para a densidade obteve efeito somente da temperatura de prensagem. Os valores médios obtidos para o teor de umidade dos compósitos prensados a $140{ }^{\circ} \mathrm{C}$ variam entre $10,45 \%$ a $11,05 \%$, não havendo diferenças estatísticas entre as médias. Já os produzidos a $160{ }^{\circ} \mathrm{C}$, os valores se encontram entre 9,53\% e 10,17\%, havendo diferenças entre as médias, sendo ainda, menores que os valores obtidos a $140{ }^{\circ} \mathrm{C}$ (Tabela 2), devido ao fato conhecido de que o painel ao ser submetido a maiores temperaturas em sua manufatura, acarretará na secagem em demasia do mesmo. Carvalho et al., (2015) encontraram um teor de umidade médio para compósitos obtidos a partir de madeira de Pinus caribaea var. hondurensis de 11,9\% e Morais et al., (2015) encontraram para compósitos de Pinus taeda valores de 12,84\%, ambos próximos aos obtidos neste trabalho.

\section{Inchamento em espessura (IE) após 2 e $24 \mathrm{~h}$}

O valor médio obtido para o IE em 2 horas foi de 19,64\% para compósitos prensados a $140{ }^{\circ} \mathrm{C}$ e de $21,58 \%$ a $160{ }^{\circ} \mathrm{C}$. O inchamento em 24 horas, tem valor médio de 26,68\% para compósitos prensados a $140{ }^{\circ} \mathrm{C}$ e $28,33 \%$ para os 
prensados a $160{ }^{\circ} \mathrm{C}$ (Tabela 3). De modo geral, o aumento da temperatura de prensagem aumenta os valores de IE, tanto após 2 horas quanto após 24 horas, isso pode ocorrer pois após abertura da prensa as tensões acumuladas (maiores conforme aumento da temperatura) são gradualmente liberadas e o uso da resina ureia-formaldeído confere baixa molhabilidade ao compósito, conforme Mohebby et al., (2009).

Tabela 2. Médias da densidade e umidade dos compósitos obtidos por tratamento analisado.

Table 2. Means of density and humidity of composites obtained by treatment analyzed.

\begin{tabular}{|c|c|c|c|c|}
\hline \multirow{2}{*}{ Teor de partícula casca (\%) } & \multicolumn{2}{|c|}{ Densidade $\left(\mathrm{g} \mathrm{cm}^{-3}\right)$} & \multicolumn{2}{|c|}{ Umidade (\%) } \\
\hline & $140{ }^{\circ} \mathrm{C}$ & $160^{\circ} \mathrm{C}$ & $140^{\circ} \mathrm{C}$ & $160^{\circ} \mathrm{C}$ \\
\hline 0 & $0,65 a^{\star 11}$ & $0,61 b^{* 18}$ & $10,62 \mathrm{a} \mathrm{AB}^{\star 2}$ & $9,68 \mathrm{~b} \mathrm{~A}^{* 2}$ \\
\hline 5 & $0,65 a^{\star 15}$ & $0,59 \mathrm{~b} * 11$ & $10,45 \mathrm{a} \mathrm{AB}^{\star 4}$ & $9,12 \mathrm{~b} \mathrm{AB}^{\star 8}$ \\
\hline 10 & $0,63 \mathrm{a}^{* 11}$ & $0,65 \mathrm{~b} * 11$ & $11,05 \mathrm{a} \mathrm{AB}^{\star 5}$ & $9,53 \mathrm{~b} \mathrm{AB}^{\star 9}$ \\
\hline 15 & $0,61 \mathrm{a}^{\star 9}$ & $0,59 \mathrm{~b} * 12$ & $10,75 \mathrm{a} \mathrm{AB}^{\star 2}$ & $9,93 \mathrm{~b} \mathrm{~B} * 4$ \\
\hline 20 & $0,60 a * 17$ & $0,54 \mathrm{~b} * 15$ & 10,76 a B ${ }^{\star 3}$ & $10,17 \mathrm{ab} \mathrm{BC}{ }^{\star 4}$ \\
\hline
\end{tabular}

Médias seguidas pela mesma letra maiúscula não diferem significativamente entre si, com relação à porcentagem de partícula casca, dentro da temperatura, pelo teste Tukey ao nível de 95\% de significância e médias seguidas pela mesma letra minúscula não diferem significativamente entre si, com relação à variação de temperatura de prensagem, pelo teste Tukey ao nível de $95 \%$ de significância. *coeficiente de variação (\%).

Tabela 3. Valores médios do inchamento em espessura após 2 e $24 \mathrm{~h}$.

Table 3. Mean values of swelling in thickness after 2 and $24 \mathrm{~h}$.

\begin{tabular}{|c|c|c|c|c|}
\hline \multirow{2}{*}{$\begin{array}{l}\text { Teor de partícula } \\
\text { casca }(\%)\end{array}$} & \multicolumn{2}{|c|}{$\begin{array}{l}\text { Inchamento em espessura após } 2 \text { horas - IE } \\
2 \mathrm{~h}(\%)\end{array}$} & \multicolumn{2}{|c|}{$\begin{array}{l}\text { Inchamento em espessura após } 24 \text { horas - IE } \\
24 \mathrm{~h}(\%)\end{array}$} \\
\hline & $140^{\circ} \mathrm{C}$ & $160^{\circ} \mathrm{C}$ & $140{ }^{\circ} \mathrm{C}$ & $160^{\circ} \mathrm{C}$ \\
\hline 0 & $19,89 \mathrm{a} \mathrm{A}^{\star 16}$ & $23,47 \mathrm{~b} \mathrm{BC}{ }^{\star 21}$ & $28,40 \mathrm{a} \mathrm{A}^{\star 16}$ & $28,98 \mathrm{~b} \mathrm{~A}^{\star 22}$ \\
\hline 5 & $18,76 \mathrm{a} \mathrm{A}^{\star 26}$ & $25,54 \mathrm{~b} \mathrm{C}^{\star 22}$ & $25,00 \mathrm{a} \mathrm{A}^{\star 18}$ & $30,05 \mathrm{~b} \mathrm{~A}^{* 19}$ \\
\hline 10 & $18,72 \mathrm{a} \mathrm{A}^{\star 20}$ & 22,31 a $\mathrm{ABC}{ }^{\star 28}$ & $26,41 \mathrm{a} \mathrm{A}^{\star 13}$ & $32,07 \mathrm{~b} \mathrm{~A}^{\star 30}$ \\
\hline 15 & $20,27 \mathrm{a} \mathrm{A}^{\star 17}$ & $19,52 \mathrm{a} \mathrm{AB}^{\star 14}$ & 26,97 a $A^{\star 17}$ & $27,01 \mathrm{~b} \mathrm{~A}^{\star 12}$ \\
\hline 20 & $20,55 \mathrm{a} \mathrm{A}^{\star 22}$ & 17,08 a $A^{\star 15}$ & 26,64 a $A^{\star 19}$ & $23,55 \mathrm{~b} \mathrm{~A}^{\star 14}$ \\
\hline
\end{tabular}

Médias seguidas pela mesma letra maiúscula não diferem significativamente entre si, com relação à porcentagem de partícula casca, dentro da temperatura, pelo teste Tukey ao nível de 95\% de significância e médias seguidas pela mesma letra minúscula não diferem significativamente entre si, com relação à variação de temperatura de prensagem, pelo teste Tukey ao nível de $95 \%$ de significância. *coeficiente de variação (\%).

Tabela 4. Valores médios de absorção de água após 2 e $24 \mathrm{~h}$.

Table 4. Mean values of water absorption after 2 and $24 \mathrm{~h}$.

\begin{tabular}{|c|c|c|c|c|}
\hline \multirow{2}{*}{$\begin{array}{l}\text { Teor de partícula } \\
\text { casca }(\%)\end{array}$} & \multicolumn{2}{|c|}{ Absorção de água após 2 horas - AA 2h (\%) } & \multicolumn{2}{|c|}{ Absorção de água após 24 horas - AA 24h (\%) } \\
\hline & $140{ }^{\circ} \mathrm{C}$ & $160{ }^{\circ} \mathrm{C}$ & $140{ }^{\circ} \mathrm{C}$ & $160^{\circ} \mathrm{C}$ \\
\hline 0 & $73,69 A^{\star 12}$ & $75,30 \mathrm{~A} * 10$ & $84,89 \mathrm{~A}^{\star 12}$ & $87,57 \mathrm{~A} * 8$ \\
\hline 5 & $74,35 \mathrm{~A} \star 20$ & $79,37 \mathrm{~A} * 9$ & $88,46 \mathrm{AB} * 18$ & $91,82 \mathrm{AB}{ }^{\star 7}$ \\
\hline 10 & $85,66 \mathrm{~A}^{\star 23}$ & $76,28 \mathrm{~A} * 22$ & $98,11 \mathrm{~B} * 19$ & $89,91 \mathrm{~B} * 18$ \\
\hline 15 & $74,55 \mathrm{~A} * 16$ & $67,08 \mathrm{~A} * 15$ & $88,76 \mathrm{~A}^{* 13}$ & $78,91 \mathrm{~A} * 11$ \\
\hline 20 & $77,94 \mathrm{~A} * 10$ & $77,41 \mathrm{~A} * 20$ & $93,30 \mathrm{AB} * 10$ & $89,15 \mathrm{AB} * 15$ \\
\hline
\end{tabular}

Médias seguidas pela mesma letra maiúscula não diferem significativamente entre si, com relação à porcentagem de partícula casca, dentro da temperatura, pelo teste Tukey ao nível de $95 \%$ de significância. ${ }^{*}$ coeficiente de variação (\%). 


\section{Absorção de água (AA) após 2 e $24 h$}

O valor médio obtido para o AA após 2 horas foi de $77,24 \%$ em compósitos prensados a $140{ }^{\circ} \mathrm{C}$ e de $75,09 \%$, naqueles fabricados a $160{ }^{\circ} \mathrm{C}$. Para a AA em 24 horas, o valor médio a $140{ }^{\circ} \mathrm{C}$ foi de $90,70 \%$ e para $160{ }^{\circ} \mathrm{C}$ o valor médio foi de $87,47 \%$ (Tabela 4). Em ambos os casos, a variação de temperatura aumenta os valores de AA obtidos tanto após 2 horas quanto 24 horas, entretanto os valores entre tratamentos não diferem estatisticamente. Pela a análise de variância da AA2h e AA24h dos compósitos produzidos foi observado que o efeito da interação foi significativo, tendo em vista que obteve efeito somente da porcentagem de casca adicionada. Rios et al., (2016), encontraram para compósitos de Pinus spp um valor médio de AA em 2 horas de 113,31\% e para AA em 24 horas de 127,06\%.

\section{Módulo de ruptura, módulo de elasticidade e}

\section{ligação interna}

A NBR 14810 (ABNT, 2013) estabelece um valor mínimo para módulo de ruptura (MOR) de $11 \mathrm{~N} \mathrm{~mm}^{-2}$ para compósitos aglomerados destinados para uso interior, sendo este valor alcançado nos tratamentos 0C/140 para compósitos prensados a $140{ }^{\circ} \mathrm{C}$ e nos tratamentos $0 \mathrm{C} / 160,5 \mathrm{C} / 160$,
$10 \mathrm{C} / 160$ e $15 \mathrm{C} / 160$ para aqueles prensados a $160{ }^{\circ} \mathrm{C}($ Tabela 5).

Pela análise de variância do MOR, MOE e LI dos compósitos, foi observado que o efeito da interação foi significativo, tendo vista que para o MOE obteve efeito somente da temperatura de prensagem e para o LI obteve efeito somente para a porcentagem de casca adicionada. O aumento da temperatura na confecção dos compósitos de $140{ }^{\circ} \mathrm{C}$ para $160^{\circ} \mathrm{C}$ promoveu uma melhora de $12 \%$ no MOR. Esse resultado mostra também que a casca exerceu, neste caso, pouca influência na resistência dos compósitos, sendo possível sua utilização em até 15\%. Batista et al., (2007) encontraram para compósitos confeccionados com 90\% de madeira de Pinus elliottii Engelm. e 10\% de casca de Eucalyptus pellita $\mathrm{F}$. Muell valor médio de $10,36 \mathrm{~N} \mathrm{~mm}^{-2}$.

Em relação ao módulo de elasticidade, a NBR 14810 (ABNT, 2013) estabelece um valor mínimo de $1.800 \mathrm{~N} \mathrm{~mm}^{-2}$ para compósitos aglomerados de uso interior, sendo alcançado somente pelo tratamento 15C/160 (Tabela 5). A temperatura também influencia o MOE, com os melhores resultados obtidos a $160{ }^{\circ} \mathrm{C}$. Batista et al., (2007), encontraram para compósitos confeccionados com $90 \%$ de madeira de Pinus elliottii Engelm. e 10\% de casca de Eucalyptus pellita $\mathrm{F}$. Muell. valor médio de 1653,90 $\mathrm{N} \mathrm{mm}^{-2}$.

Tabela 5. Valores médios das propriedades mecânica dos compósitos (Módulo de Ruptura MOR; Módulo de Elasticidade MOE e Ligação Interna - LI).

Table 5. Mean values of mechanical properties of composites (MOR Breakage Modulus, Elasticity Modulus - MOE and Internal Bonding - LI).

\begin{tabular}{|c|c|c|c|c|c|c|}
\hline \multirow{2}{*}{$\begin{array}{c}\text { Teor de partícula } \\
\text { casca }(\%)\end{array}$} & \multicolumn{2}{|c|}{$\operatorname{MOR}\left(\mathrm{N} \mathrm{mm} \mathrm{mm}^{-2}\right)$} & \multicolumn{2}{|c|}{$\operatorname{MOE}\left(\mathrm{N} \mathrm{mm} \mathrm{mm}^{-2}\right)$} & \multicolumn{2}{|c|}{ LI $\left(\mathrm{N} \mathrm{mm}^{-2}\right)$} \\
\hline & $140^{\circ} \mathrm{C}$ & $160^{\circ} \mathrm{C}$ & $140^{\circ} \mathrm{C}$ & $160^{\circ} \mathrm{C}$ & $140^{\circ} \mathrm{C}$ & $160^{\circ} \mathrm{C}$ \\
\hline 0 & 12,17 a $A^{\star 22}$ & $11,47 \mathrm{~b} \mathrm{~A}^{\star 24}$ & $1699,98 a^{\star 28}$ & $1795,35 b^{\star 28}$ & $0,41 \mathrm{AB} * 34$ & $0,47 \mathrm{AB} * 25$ \\
\hline 5 & $10,82 \mathrm{a} \mathrm{A}^{\star 26}$ & $11,63 \mathrm{~b} \mathrm{~A}^{\star 18}$ & $1595,38 \mathrm{a}^{\star 19}$ & $1591,89 b^{\star 20}$ & $0,45 \mathrm{~A}^{\star 18}$ & $0,53 A^{\star 29}$ \\
\hline 10 & $10,20 \mathrm{a} \mathrm{A}^{\star 23}$ & $11,98 \mathrm{~b} \mathrm{~A}^{\star 21}$ & $1507,43 \mathrm{a}^{\star 27}$ & $1772,53 b^{\star 22}$ & $0,47 A^{\star 29}$ & $0,54 \mathrm{~A}^{\star 27}$ \\
\hline 15 & $8,16 \mathrm{a} \mathrm{AB}^{\star 21}$ & $11,37 \mathrm{~b} \mathrm{~A}^{\star 13}$ & $1341,63 \mathrm{a}^{\star 16}$ & $1937,71 b^{\star 12}$ & $0,47 \mathrm{AB} * 13$ & $0,41 \mathrm{AB}{ }^{\star 35}$ \\
\hline 20 & $6,90 \mathrm{a} \mathrm{B}^{\star 17}$ & $8,43 \mathrm{~b} \mathrm{~B}^{\star 31}$ & $1202,32 \mathrm{a}^{* 17}$ & $1571,58 b^{\star 27}$ & $0,38 \mathrm{~B}^{* 17}$ & $0,39 \mathrm{~B} * 27$ \\
\hline
\end{tabular}

Médias seguidas pela mesma letra maiúscula não diferem significativamente entre si, com relação à porcentagem de partícula casca, dentro da temperatura, pelo teste Tukey ao nível de 95\% de significância e médias seguidas pela mesma letra minúscula não diferem significativamente entre si, com relação à variação de temperatura de prensagem, pelo teste Tukey ao nível de 95\% de significância. * coeficiente de variação (\%). 
Já para a ligação interna ou resistência à tração perpendicular, a NBR 14810 (ABNT, 2013) estabelece um valor de $0,40 \mathrm{~N} \mathrm{~mm}^{-2}$ para compósitos aglomerados de uso interior, sendo alcançados por todos os tratamentos, a excessão dos tratamentos com adição de $20 \%$ de casca. Houve uma influência da adição da casca sobre a resistência a tração dos compósitos, havendo melhora de mais de $12 \%$ para os tratamentos $10 \mathrm{C} / 140, \quad 15 \mathrm{C} / 140$ e $10 \mathrm{C} / 160$. Os valores obtidos foram superiores ao alcançado por Batista et al., (2007) de 0,19 $\mathrm{N} \mathrm{mm}^{-2}$ para compósitos com 90\% de Pinus elliottii Engelm. e 10\% de casca de Eucalyptus pellita F. Muell.

\section{pH, capacidade tampão e concentração de}

\section{formaldeído}

Observa-se pela Tabela 6 que a medida que se aumenta o percentual de casca nos diferentes compósitos produzidos, possibilita uma redução gradativa nos valores de $\mathrm{pH}$, tornando-os mais ácidos. Este fato pode estar associado ao teor de extrativos encontrados na casca, que é mais elevado que na madeira, e, portanto, contribui para um $\mathrm{pH}$ mais ácido (TRIANOSKI et al., 2013). O valor médio do $\mathrm{pH}$ para a temperatura de $140{ }^{\circ} \mathrm{C}$ foi de 1,48 e já para a temperatura de

$160{ }^{\circ} \mathrm{C}$ foi de 1,40 . Em relação a capacidade tampão o valor médio para ambas temperaturas foi de $0,11 \mathrm{mmol} \mathrm{NaOH}$ $100 \mathrm{~g}^{-1}$ material seco, devido ao baixo $\mathrm{pH}$.

Pela a análise de variância do $\mathrm{pH}$, capacidade tampão e concentração de formaldeído dos compósitos, foi observado que o efeito da interação foi significativo, tendo visto que para o $\mathrm{pH}$ obteve efeito somente para a porcentagem de casca adicionada. A faixa de concentração de formaldeído obtida foi de $0,0075 \mathrm{mg} \mathrm{L}^{-1}$ a $0,0116 \mathrm{mg} \mathrm{L}^{-1}$ para os tratamentos realizados

Pode-se verificar, nos compósitos confeccionados a 140 ${ }^{\circ} \mathrm{C}$, que houve uma atenuação da concentração de formaldeído no tratamento $0 \mathrm{C} / 140$, seguido de uma elevação para o tratamento $10 \mathrm{C} / 140$ e depois uma redução até o último tratamento 20C/140. Para os compósitos confeccionados na temperatura de $160{ }^{\circ} \mathrm{C}$, observou-se um aumento da concentração de formaldeído para os tratamentos 0C/160 e 5C/160 e uma redução gradual para os demais tratamentos (Figura 1). Zhang et al., (2015) obtiveram em painéis de partículas uma concentração média de formaldeído de $0,014 \mathrm{mg} \mathrm{L}^{-1}$, superior a encontrada neste trabalho. Destarte, este trabalho confere uma redução

Tabela 6. Valores médios de $\mathrm{pH}$, capacidade tampão e da concentração de formaldeído dos compósitos por tratamento realizado.

Table 6. Mean values of $\mathrm{pH}$ and buffer capacity and of formaldehyde concentration of composites per treatment performed.

\begin{tabular}{ccccccc}
\hline \multirow{2}{*}{$\begin{array}{c}\text { Teor de partícula } \\
\text { casca (\%) }\end{array}$} & \multicolumn{2}{c}{$\mathbf{p H}$} & \multicolumn{2}{c}{$\begin{array}{c}\text { Capacidade Tampão (mmol NaOH } \\
\mathbf{1 0 0 g}^{-1} \text { material seco) }\end{array}$} & \multicolumn{2}{c}{$\begin{array}{c}\text { Concentração de } \\
\text { formaldeído (mg L }\end{array}$} \\
\cline { 2 - 7 } )
\end{tabular}

Médias seguidas pela mesma letra maiúscula não diferem significativamente entre si, com relação à porcentagem de partícula casca, dentro da temperatura, pelo teste Tukey ao nível de 95\% de significância e pelo teste não paramétrico Kruskal-Wallis para a capacidade tampão, e médias seguidas pela mesma letra minúscula não diferem significativamente entre si, com relação à variação de temperatura de prensagem, pelo teste Tukey ao nível de $95 \%$ de significância significância e pelo teste não paramétrico Kruskal-Wallis para a capacidade tampão. * coeficiente de variação (\%). 
considerável na emissão de formaldeído nos compósitos produzidos a $140{ }^{\circ} \mathrm{C}$ com $15 \%$ e $20 \%$ de casca adicionada, sendo esta redução de $18 \%$ e $35 \%$, respectivamente, com relação ao produzido sem casca adicionada. E este fato ocorre devido sobretudo a compostos fenólicos presentes na casca que reagem com o formaldeído livre nos compósitos, levando à diminuição nos teores de emissão.

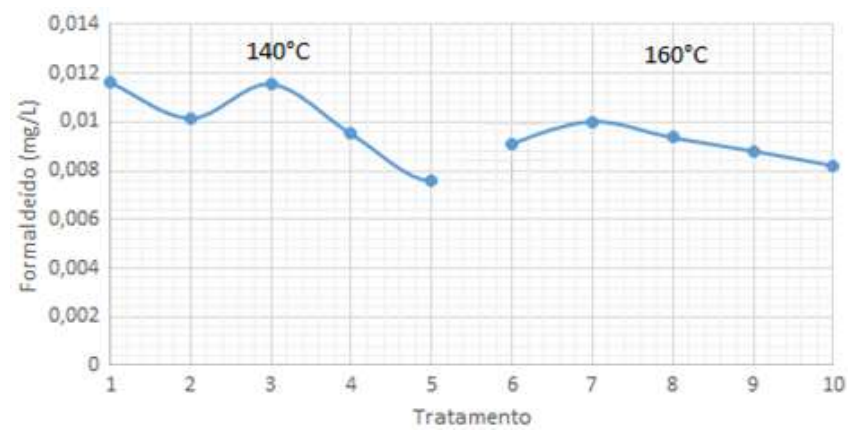

Figura 1. Redução da concentração de formaldeído em função do tratamento realizado.

Figure 1. Reduction of formaldehyde concentration as a function of treatment performed.

\section{Conclusões}

Por todo o exposto, denota-se do presente estudo a viabilidade dos compósitos produzidos a partir de Pinus caribaea var. caribaea (sob diferentes proporções de madeiracasca) e que sobrevém uma redução da emissão de formaldeído à medida que se aumenta a proporção de partícula casca. As diferentes proporções realizadas influenciam positivamente nas propriedades dos compósitos, não só na redução da emissão de formaldeído como também no reaproveitamento da casca, com o melhor resultado obtido para $15 \%$ de casca à $160^{\circ} \mathrm{C}$.

\section{Referências}

ASSOCIAÇÃO BRASILEIRA DE NORMAS TÉCNICAS ABNT. NBR 14810: Chapas de madeira aglomerada. Rio de Janeiro, 2013.

BATISTA, D. C. et al. Fabricação de aglomerados de três camadas com madeira de Pinus elliottii Engelm. e casca de
Eucalyptus pellita F. Muell. Cerne, Lavras, v. 13, n. 02, p. 178$187,2007$.

AMERICAN NATIONAL STANDARDS INSTITUTE ANSI. ANSI-A-208.1: Mat-formed wood particleboard. New York, 1987.

BRITO, E. O. et al. Chapas de madeira aglomerada de uma camada de Pinus elliottii Engelm. com a adição das cascas de Eucalyptus pellita f. Muell. Cerne, Lavras, v. 11, n. 04, p. 369375, 2005.

CAMPOS, C. I.; LAHR, F. A. R. Estudo comparativo dos resultados de ensaio de tração perpendicular para MDF produzido em laboratório com fibras de pinus e de eucalipto utilizando uréia-formaldeído. Matéria, Rio de Janeiro, v. 09, n. 01, p. 32-42, 2004.

CARVALHO, A. G. et al. Efeito da adição de resíduos de poda da erva-mate em painéis aglomerados. Revista Árvore, Viçosa, v.39, n.1, p.209-214, 2015.

DAI, X. et al. Modeling and controlling indoor formaldehyde concentrations in apartments: On-site investigation in all climate zones of China. Building and Environment, v. 127, p. 98-106, 2018.

EUROPEAN STANDARD - EN. EN 717-3: Wood-based panel products - Determination of

formaldehyde release by the flask method. 1996.

FURTADO, T. S. et al. Variáveis do processo de briquetagem e qualidade de briquetes de biomassa florestal. Pesquisa Florestal Brasileira, Colombo, v. 30, n. 62, p. 101-106, 2010.

HE, Z.; ZHANG, Y.; WEI, W. Formaldehyde and VOC emissions at different manufacturing stages of wood-based panels. Building and Environment, v. 47, p. 197-204, 2012.

INTERNATIONAL AGENCY FOR RESEARCH ON CANCER - IARC. Formaldehyde, 2-butoxyethanol and 1tert-butoxypropan-2-ol. v. 88. Lyon: IARC, 2006. 478p.

JANTUNEN, M. et al. Indoor air pollution exposure. Indoor Air Quality and Health Effects. Projeto n. SSPE-CT-2004502671, 2008.

MALONEY, T. M. Modern particleboard and dry-process fiberboard manufacturing. 2. ed. San Francisco: Miller Freeman, 1993. 689 p. 
MESQUITA, R. G. A. et al. Inclusão de feixes de sisal na produção de painéis MDP de eucalipto. Scientia Forestalis, v. 43, n. 105, 2015.

MOHEBBY, B, et al. Springback in acetylated wood based composites. Construction and Building Materials, v. 23, n. 9, p. 3103-3106, 2009.

MORAIS, W. W. C. et al. Propriedades físico-mecânicas de painéis aglomerados com Bambusa tuldoides e Pinus taeda. Ciência Florestal, Santa Maria, v. 25, n. 4, p. 1015-1026, 2015.

PEREIRA, E. A. et al. Gota suspensa para avaliação de aldeído total no ar interno e externo do ambiente. Química Nova. v. 24, n. 4, p 443-448, 2001.

PIERCE, J. S. et al. An assessment of formaldehyde emissions from laminate flooring manufactured in China. Regulatory Toxicology and Pharmacology, v. 81, p. 20-32, 2016.

RIOS, P. D. et al. A. Avaliação do potencial da madeira de Pinus patula Schltdl. \& Cham para a produção de painéis de madeira aglomerada. Scientia Forestalis, Piracicaba, v. 44, n. 110, p. 497-508, 2016.

TRIANOSKI, R. et al. Propriedades físicas e mecânicas de painéis de madeira aglomerada de Acrocarpus fraxinifolius, compostos com diferentes percentuais de casca. Ciência Florestal, Santa Maria, v. 23, n. 04, p. 761-769, 2013.

VITAL, B. R. Método de determinação da massa específica da madeira. Viçosa: Boletim Técnico - SIF, n. 2. 1984. 21p.

ZHANG, Y. et al. Urea formaldehyde resin with low formaldehyde content modified by phenol formaldehyde intermediates and properties of its bamboo particleboards. Journal of Applied Polymer science, v. 132, 27, 2015. 